\title{
Competencias personales docentes para la educación inclusiva: una reflexión sobre lo que está ocurriendo en España
}

Eladio Sebastián-Heredero*

\section{Resumo}

Desde el final del siglo pasado España viene desarrollando e implementando sus políticas de inclusión educativa. Es ahora un buen momento para hacer una reflexión de las lecciones aprendidas sobre las variables personales que el docente de la educación inclusiva debe tener desde tres perspectivas: su formación inicial, las que exige de la formación continuada en servicio y las que proceden de su práctica docente. El profesor actual precisa tener como profesional un conjunto de competencias entendidas como los recursos - conocimientos, habilidades y actitudes - que necesita para resolver de forma satisfactoria las situaciones a las que se enfrenta en su trabajo: planificar, crear y reconstruir nuevas formas de motivar y enseñar a todos; compartir conocimiento y trabajar de forma colaborativa; pero también ser líder, escuchar, usar Tecnologías de Información y Comunicación o ser miembro de un equipo y de la comunidad. Competencias cognitivas y no cognitivas entran en juego en este ensayo.

Palavras-chave: Educación inclusiva; Competencias docentes; Políticas educativas.

\footnotetext{
* Professor doutor da Universidad de Alcalá, Alcalá de Henares, Madrid, Espanha.
} 


\section{Personal teaching competencies for inclusive education: a reflection on what is occurring in Spain}

\section{Abstract}

Since the end of the last century Spain has been developing and implementing its educational inclusion policies. It is now a good time to reflect on the lessons learned about the personal variables that the teacher of inclusive education must have from three perspectives: his initial training, those that require continuing education in service and those that come from his teaching practice. The current teacher needs to have as a professional a set of skills understood as the resources - knowledge, skills and attitudes - that he needs to satisfactorily solve the situations he faces in his work: planning, creating and rebuilding new ways of motivating and teach everyone; share knowledge and work collaboratively; but also be a leader, listen, use Information and Communication Technologies or be a member of a team and community. Cognitive and non-cognitive competencies come into play in this essay.

Keywords: Inclusive education; Teacher competencies; Educational policies.

\section{Un poco de historia}

España desde la década de los 80 del siglo XX, ha sufrido enormes transformaciones en materia educativa que comenzaron por los Pactos de la Moncloa en los ańos posteriores a la finalización de la dictadura y que tuvo su punto más álgido en la publicación de la Ley Orgánica General del Sistema Educativo (LOGSE) en el año 1990 (ESPAÑA, 1990). Primera y única ley de educación a la que se llegó por consenso de todos los grupos políticos de la época y que sirvió de referente para un cambio conceptual de entender la educación que no sólo fue un referente para todo lo que viene ocurriendo en España desde entonces, sino para otros muchos países, como Brasil, que la tomaron como referencia para organizar sus propias políticas educativas.

La LOGSE llevó consigo, entre otras cosas, la extensión de la educación obligatoria y gratuita de los 6 a los 16 años, lo que implicó la introducción de la educación inclusiva pues esta atención se daba en situación de igualdad de acceso y formación para todos los educandos dentro de una concepción de currículo comprehensivo y sin diferencias con las adecuaciones que fueran necesarias para poder atender a todos estos alumnos incluso con medidas como la opcionalidad, la transversalidad, el programa diversificado o las adecuaciones curriculares. Pero además una mejora de la carrera docente que se materializó en varios aspectos como el cambio en la formación inicial del profesorado, la creación de los Centros de Formación Permanente del Profesorado, las mejoras profesionales y salariales, la creación de proyectos educativos de centros que permitían el trabajo en equipo y, concomitantemente, la necesidad de adquirir nuevas competencias que le permitiesen atender esta nueva realidad que superaba el simple hecho de enseñar trasladándolo a la concepción de educar en sentido amplio pasando aquella famosa sentencia, que solo conduce al fracaso escolar: morir de pie, con una tiza en la mano, en la pizarra de Huberman (1989). 
La configuración del sistema educativo español fue totalmente modificada con la creación de dos tipos de instituciones: los Colegios de Educación Infantil y Primaria (CEIP) para la atención de alumnos de los 3 a los 12 ańos de edad y los Institutos de Educación Secundaria (IES) para alumnos de 12 a 18 años.

La etapa educativa de educación infantil tiene tres cursos de duración, de los 3 a los 6 años, es de oferta obligatoria para las administraciones educativas que han de garantizar plazas para todos los alumnos y de libertad para las familias que pueden optar por llevarlos a la escuela o educarlos en casa, tiene como objetivo la socialización y el desarrollo de habilidades básicas, son atendidos por un profesor especialista en Educación Infantil. La etapa de educación primaria es obligatoria para todos los alumnos y tiene seis cursos de duración entre los 6 y los 12 años de edad, con la posibilidad de poder repetir tan solo un año en toda la etapa. En esta etapa el currículo es comprehensivo, es decir para todos los alumnos igual a partir de disciplinas y tiene como objetivo formar en las competencias instrumentales básicas que les permitan continuar con éxito su proceso de aprendizaje, son atendidos por un profesor generalista o maestro que realiza además la función de tutor del grupo ya que estos alumnos, además de este profesor, tienen clases con especialistas como Inglés, Música o Educación Física.

La etapa educativa de Secundaria tiene dos tramos bien diferenciados. La educación secundaria obligatoria (ESO) que está organizada en cuatro años escolares desde los 12 a los 16 años y que ha variado de su configuración inicial de currículo comprehensivo y con cierto carácter tecnológico hacia una configuración de currículo diversificado en función del rendimiento de los alumnos y segregador, pues en el tercer año ya hay dos opciones una pre-profesional y otra académica y en cuarto año tres opciones. Está organizado de forma tradicional en disciplinas que son atendidas por profesores con graduación en esa especialidad y un máster en educación que los habilita específicamente para la docencia. Tras esta etapa obligatoria, que puede acabar a partir de los 16 años, los alumnos tienen tres opciones abandonar el sistema educativo o seguir en la formación reglada ya sea en el Grado Medio de Formación Profesional (CFGM) o Bachillerato. Los CFGM de uno o dos años de duración son de carácter profesionalizante y los habilita para cursar un Grado Superior de Formación Profesional (CFGS), incorporarse al mundo laboral o cursar estudios de Bachillerato. El Bachillerato tiene dos años de duración, se organiza en tres grandes áreas de conocimiento: Humanas, Ciencias de la Salud y Ciencias Tecnológicas y los habilita para acceder a la Universidad.

Durante todas las etapas educativas obligatorias: Educación Infantil, Educación Primaria y Educación Secundaria Obligatoria, las familias pueden incorporar a sus hijos e hijas a la educación ordinaria, aunque existen centros de Educación Especial para los casos más graves. Estos centros ordinarios se deben encontrar en condiciones de atender a cualquier tipo de alumno con cualquier tipo de característica para lo que deben elaborar un Plan de Atención a la Diversidad que regule la atención de todos ellos a partir de un diagnóstico elaborado en la propia escuela que determinará las necesidades educativas ${ }^{1}$ que pueda precisar y la atención específica para su caso en concreto que incluye personal y medidas. 
En materia de educación inclusiva los avances de aquella legislación nacional se concretaron en personal específico de atención al alumnado y de medidas concretas de respuesta a la diversidad, con legislación nacional que desarrolló de forma concreta y específica sus funciones y acciones que después seria desarrollada a posteriori por las diferentes Comunidades Autónomas $(\mathrm{CCAA})^{2}$ en la medida que ellas consideraron conveniente.

Entre los modelos específicos de atención al alumnado para todos los centros educativos fue incorporada la presencia de profesores de enseñanza básica de Pedagogía Terapéutica (PT) y de Audición y Lenguaje (AL), además de otros profesionales que podrían participar en la atención a alumnos con necesidades educativas especiales como eran los Fisioterapeutas o los Auxiliares Técnicos Sanitarios, incluso la dotación en centros ordinarios de un profesional de la educación especial que era el Auxiliar Técnico Educativo. Esta nueva dotación supuso que el maestro titular de casa una de las clases, como dijimos anteriormente tutor, deba empezar a tener otro tipo de formación y de competencias que le permitan trabajar con esa realidad.

Para organizar la atención educativa del alumnado con necesidades educativas dentro de ese contexto diferente de escuelas inclusivas dentro del marco del plan de atención a la diversidad, la administración educativa hizo posible que todos los centros de educación infantil y primaria y todos los institutos de secundaria tuviesen un Orientador, profesional de la psicología, pedagogía o psicopedagogía, con la responsabilidad de realizar los informes psicopedagógicos de los alumnos en los que se fijan las necesidades educativas de los alumnos y en función de ellas la forma de atención y de escolarización. En la forma de atención a estos alumnos se determinan las orientaciones para la realización de las adaptaciones o adecuaciones curriculares necesarias y las orientaciones de cómo tratar y como trabajar con ese alumno tanto para el profesor tutor como para los profesores especialistas de PT y AL.

\section{Necesidades formativas del profesorado derivadas del nuevo contexto educativo}

Esta nueva ordenación del sistema educativo y todas las medidas que llevó aparejadas hizo necesaria una serie de cambios tanto en la forma de formar a los profesores como en el qué formar a los profesores Soriano y Vigo (2008). No cabe duda de que los profesores necesitaban nuevas competencias que dieran respuesta al modelo inclusivo introducido y a la forma de atender a los alumnos en las clases ordinarias por los profesores tutores, ya decía Meirieu (1992) estamos antes un escenario para un nuevo oficio.

En lo referente a la formación inicial del profesorado la introducción de formaciones específicas y especializadas tanto del maestro de PT como de AL, pues era en primer lugar un profesor que podía asumir un grupo de alumnos ordinarios. Estos especialistas deben trabajar junto con el profesor tutor en la atención de los alumnos con necesidades educativas especiales, lo que implica que el profesor tutor ha de aprender a trabajar de forma diferenciada con este tipo de alumnos al mismo tiempo que con todos. La inclusión en los cursos de graduación de disciplinas como educaci- 
ón inclusiva, medidas de atención a las necesidades educativas, metodologías de aula, tecnologías de la educación, etc., mucho ayudaron en este proceso.

Pero eso no era suficiente y los Centros de Formación Continuada de Profesores, creados por el Ministerio de Educación para facilitar la formación de los docentes en activo, programaron y desarrollaron múltiples acciones en sus diferentes formatos de Cursos, Seminarios y Grupos de Trabajo para mejorar la competencia docente en ese y en otros muchos campos de la educación.

Y además de estas dos acciones todavía muchos centros, tanto de primaria como de secundaria, ensayaron modelos y acciones para atender mejor a sus alumnos a partir del Plan de Atención a la Diversidad que pasó a formar parte del Proyecto Educativo y que era el instrumento para dar cobertura organizativa a la atención a todos los alumnos de las escuelas. La organización de medidas de apoyo y refuerzo, de adaptaciones curriculares, de programas de diversificación curricular, de agrupamientos flexibles, de metodologías activas, etc., fueron incorporadas a la práctica para unos profesores para los que lo más importante era educar más que enseñar.

En otro orden de cambios de contexto que modificaron la forma de pensar de los profesores y en consecuencia sus formas de trabajar tuvieron que adaptarse fue la explosión económica de España a partir del año 1996. Este hecho acarreó el aumento de la población escolar por la llegada de inmigrantes, con la creación de nuevos centros para poder acogerlos; pero al mismo tiempo la necesidad de diseñar nuevas formas de trabajar con estos alumnos, que venían de unas condiciones muy distintas de aprendizaje y cultura y que de repente se encontraron en la misma clase con una lengua vehicular que les era del todo extraña. La atención a alumnos de otras culturas conllevó también una acción del gobierno de dotación de recursos personales, el profesor de interculturalidad, un maestro o maestra con un curso de especialización de un año sobre todo en enseñanza de español como lengua extrajera y metodologías de aula. Su función también era ayudar al profesor titular del aula y atender en algunos horarios específicos a los alumnos. Pero la responsabilidad principal caía sobre el profesor tutor que debía impartir docencia en clase ahora más diversas, donde los alumnos con necesidades educativas derivadas de alguna deficiencia pasaron a ser una anécdota frente a la cantidad de alumnos de culturas y lenguas diferentes en las aulas. Lo que nuevamente hizo repensar lo que un profesor debería ser incrementándose de nuevo la formación en competencias metodológicas activas y personalizadas que diesen los resultados específicos.

Por último, no podemos dejar de hacer referencia, en este repaso, al hecho de las transferencias educativas en etapas anteriores a la Universidad a las Comunidades Autónomas que se produjo en el año 2000. Este hecho fue más importante de lo que parece pues desde las nuevas administraciones educativas, verdaderos Ministerios de Educación en cada una de ellas, se paso a legislar, ordenar y ejecutar diferentes formas de entender la educación en función del signo político de ese gobierno y por tanto desde propuestas neoliberales a las más progresistas. Eso implicó también un cambio en las competencias docentes pues coexistían dos tipos de acciones muy diferentes que implicaban trabajos diferentes de los docentes. Sirva como ejemplo que en unas 
Comunidades Autónomas existían aulas de enlace, segregadoras y excluyentes, donde los alumnos de otras culturas eran separados durante un periodo de tiempo no específico hasta el aprendizaje de la lengua española, mientras que en otras cabía la responsabilidad al equipo de profesores de la escuela organizarse y desarrollar medidas que dentro de la propia aula, con el resto de sus compańeros, estos alumnos con dos profesores, con metodologías lúdicas, con trabajos específicos, con la ayuda de iguales, etc., y guiados por el profesor tutor aprendiesen la lengua española.

\section{Las competencias docentes para el siglo XXI}

Si hacemos una revisión de la literatura observamos que son muchos los autores que indican diferentes competencias que debería tener el docente actual como consecuencia de los cambios que está sufriendo la educación (ALEGRE, 2010; FERNÁNDEZ BATANERO, 2013; TIMPERLEY; ALTON-LEE, 2008; ZABALA; ARNAU, 2007).

Buscando un concepto consensuado diremos que competencia consiste en una capacidad de movilizar varios recursos cognitivos para hacer frente a algún tipo de situaciones. Esta definición, siguiendo a Le Boterf (1995) tiene cuatro componentes a ser considerados: Las competencias no son en sí mismas conocimientos, habilidades o actitudes, pero si que precisan de ellos ya que los movilizan o integran. Esta movilización sólo resulta pertinente en un determinado y único contexto, lo bueno es que se pueden utilizar o integrar en otras situaciones parecidas. Poner en funcionamiento una competencia pasa por operaciones mentales complejas, que permiten realizar una acción adaptada a cada contexto. Las competencias profesionales se crean con formación; pero también con entrenamiento y práctica.

Parece procedente comenzar este debate sobre las competencias docentes para el siglo XXI con las aportaciones de Perrenoud (2004) cuando habla de las diez grandes familias de competencias, dejando fuera las que son obvias derivadas de la necesidad de ser un experto en su disciplina, que resumidas son:

1. Organizar y animar situaciones de aprendizaje.

2. Gestionar la progresión de los aprendizajes.

3. Elaborar y hacer evolucionar dispositivos de diferenciación.

4. Implicar a los alumnos en sus aprendizajes y en su trabajo.

5. Trabajar en equipo.

6. Participar en la gestión de la escuela.

7. Informar e implicar a los padres.

8. Utilizar las nuevas tecnologías.

9. Afrontar los deberes y los dilemas éticos de la profesión.

10. Organizar la propia formación continua. 
Estas son desarrolladas en su obra "diez nuevas competencias para enseñar"; pero lo que ahora interesa es determinar si realmente coincidimos en que esto es básico para el docente pensando en él como mediador y como gestor del conocimiento. Desde una óptica de profesionalización docente no hay la menor duda de que, desde su aparición, fueron tomadas como referencias para la mayoría de los autores y discutidas en la Academia de forma profunda, ahora bien desde una perspectiva de escuela inclusiva hay que profundizar un poco más para determinar si son básicas, ante lo que nos parece evidente que estas serían incompletas después de las lecciones aprendidas.

Hacemos nuestras las palabras de Bachelard (1996) cuando observa que a los profesores les cuesta entender que sus alumnos no comprenden, puesto que han olvidado el camino del conocimiento y en ese caso hay que ponerse en el lugar de los alumnos.

Perez (2005) establece varias competencias docentes que las agrupa en torno a cinco grandes ejes que son: Comunicacionales; Organizativas; de Liderazgo Pedagógico; Científicas y de Evaluación y Control y algunas de estas competencias coinciden con las ya comentadas de Perrenoud; no obstante se destaca un grupo de competencias que podríamos llamar creativas o innovadoras, muy importantes para el docente actual, entre las que destacamos: aplicación de recursos innovadores; formación en contenidos científicos, didácticos y metodológicos o realización de proyectos innovadores, algo muy necesario en el docente desde la perspectiva inclusiva.

De otro lado Ayala (2008) habla de cinco grandes competencias todas ellas muy ligadas a procesos: Diseñador de escenarios, procesos y experiencias de aprendizaje significativos; Experto en su disciplina académica; Facilitador y guía de un proceso de aprendizaje centrado en el desarrollo integral del alumno; Evaluador del proceso de aprendizaje del alumno y responsable de la mejora continua de su curso y, para finalizar, un docente consciente y activo en el constante proceso de cambio en educación y en un contexto intercultural.

Para concluir cuáles de ellas son las más adecuadas deberíamos de realizar un análisis profundo para determinar las que se consideren necesarias y eso significa, de un lado, relacionar cada una de ellas con un grupo delimitado de problemas y tareas y, de otro lado, clasificar los conocimientos, técnicas, habilidades, aptitudes y competencias movilizados por la competencia considerada. Eso significa, a partir de las tres clasificaciones presentadas, que todas valen, pues dependen del foco y de la forma de entender la educación. Eso hace las competencias docentes algo abstracto y todavía no consensuado; pero que de alguna forma abordan tres grandes cuestiones que el profesor debe dominar: el conocimiento de su disciplina y las metodologías que permitan que los alumnos aprendan; la gestión amplia de procesos que consigan crear unas instituciones y unas aulas con un proceso de enseńanza-aprendizaje eficaz y participativo y, por último, la innovación y la creatividad que hagan del docente un sujeto que se adapta y se forma constantemente en función de las necesidades que su contexto le demanda, con la presencia de competencias cognitivas y no cognitivas. 


\section{Las competencias del profesorado para una escuela inclusiva}

Adentrándonos un poco más en las competencias docentes, ahora desde la perspectiva de una escuela inclusiva tenemos que tomar como referencia a Ainscow que junto a Booth crearon el Index para la Inclusión (BOOTH; AINSCOW, 2000) y dentro de él se trabajan tres dimensiones divididas en dos categorías, a saber: Dimensión A. Crear culturas inclusivas: construir comunidad y establecer valores inclusivos. Dimensión B. Elaborar políticas inclusivas: desarrollar una escuela para todos y organizar el apoyo para atender a la diversidad. Dimensión C. Desarrollar prácticas inclusivas: orquestar el aprendizaje y movilizar recursos. Aunque el Index no sea un instrumento para poner en cuestión las competencias profesionales del docente, pues es un modelo de apoyo a los centros educativos y para la mejora profesional de sus profesionales, no nos cabe duda que puede ilustrar en lo que eso significa definición de competencias docentes, así aparecen implícitas las competencias como: trabajar en equipo, gestionar centro y aula, gestionar el aprendizaje, elaborar propuestas innovadoras y creativas, movilizar recursos, entre otras.

Alegre (2010) describe diez capacidades docentes fundamentales para la atención a la diversidad del alumnado: capacidad reflexiva, medial, la de gestionar situaciones diversas de aprendizaje en el aula, la de ser tutor y mentor, la de promover el aprendizaje cooperativo y entre iguales, la capacidad de comunicarse e interactuar, la capacidad de proporcionar un enfoque globalizador y metacognitivo, la de enriquecer actividades de enseñanza-aprendizaje, la de motivar e implicar con metodología activas al alumnado y la de planificar.

En una investigación de campo realizada en España y coordinada por la Universidad de Granada, Fernández Batanero (2013) llega a colocar como resumen de ella que, según los profesores, habría seis grandes competencias que el docente inclusivo debería poseer: Tutorización, que vinieron a conceptualizar como una ayuda continua y dinámica de acompańamiento en la formación y educación del estudiante; Comunicación, entendida como capacidad de escuchar, hacer preguntas, expresar conceptos e ideas de forma efectiva; Gestión de metodologías activas y más específicas para este tipo de alumnos; Gestión de metodologías activas, y de forma concreta sobre aquellas que son consideradas más específicas y que capacitan al individuo, sea cual sea su condición, ante el currículo y desarrollan su autonomía general; Acomodar y ajustar cuanto sea necesario la enseńanza y aprendizaje a todos los alumnos, como condición para una educación de calidad; Gestionar la atención a las familias, como coparticipes de la educación y socios en la empresa de educar.

Por último queremos traer la referencia de una recopilación de lo que sería el perfil del docente inclusivo, creado por la Agencia Europea para el Desarrollo de la Educación del Alumnado con Necesidades Educativas Especiales (AGENCIA EUROPEA PARA EL DESARROLLO DE LA EDUCACIÓN DEL ALUMNADO CON NECESIDADES EDUCATIVAS ESPECIALES, 2012) en el que se destacan cuatro grandes competencias, unas de tipo de tipo cognitivo: Trabajar en equipo: la colaboración y el trabajo en equipo son un enfoque esencial para todos los docentes 
y desarrollo profesional permanente del profesorado: la docencia es una actividad de aprendizaje y los docentes aceptan la responsabilidad de aprender a lo largo de toda su vida; pero también de tipo no cognitivo: Valorar la diversidad del alumnado: las diferencias entre estudiantes son un recurso y un valor educativo y apoyar a todo el alumnado: los docentes han de esperar lo mejor de todos sus alumnos.

\section{Consideraciones finales}

A lo largo de estos últimos veintisiete años los cambios habidos en la educación espańola han sido muy grandes y, en algunos momentos, nos han colocado como referencia en educación inclusiva. Estos cambios no habrían sido posibles sin una profesionalización docente y la incorporación de nuevas competencias que les permitan afrontar la realidad de las aulas cada día tanto de tipo cognitivo como no cognitivo.

Las competencias que hemos visto como más importantes en la literatura se sienten en el profesorado espańol, así entendemos que estas serían: planificador; trabajar en equipo; gestor del aprendizaje; innovador; abierto a aprendizajes y cambios.

Empezamos por la capacidad de planificar, que también puede ser entendida como gestor del centro y del aula, pues se tiene consciencia de la importancia de establecer nuevas formas participativas de organización en los centros educativos desde una visión global, que en Espańa ha sido conducida por los Proyectos de Atención a la Diversidad, además de la comunicación y de su canalización para que llegue a todos.

Cuando hablamos de trabajar en equipo, ya no cabe la menor duda a todos los profesionales que el trabajo entre orientador, profesor de pedagogía terapéutica y profesor titular de clase cuando hablamos de alumnos con necesidades educativas especiales es imprescindible, desde la realización del diagnóstico, pasando por las adaptaciones, siguiendo por el desarrollo de la misma en un proceso de enseńanza -aprendizaje diferenciado pero junto al resto de los compańeros y acabando con la evaluación y decisiones de promoción de estos sujetos.

En el momento en el proponemos una gestión del aprendizaje, de una forma amplia, nos estamos refiriendo a la importancia que tiene en estos momentos desarrollar metodologías activas en el aula, con la incorporación de nuevos recursos y materiales, pero sobre todo partiendo de la base de que las aulas son muy diversas y multiculturales, en las que el profesor ha llegado a entender que todos pueden aprender de todos y con todos y no se limita a la relación profesor alumno, sino que otros actores pueden intervenir, como de hecho ocurre, familias, alumnos, otros profesionales, otros profesores. La motivación y la mejora de la autoestima son dos elementos que forman parte de las competencias no cognitivas esenciales para el buen funcionamiento de estas propuestas inclusivas de trabajar.

No cabe duda que el profesor ha de ser innovador y prueba de ello son las diferentes iniciativas que los centros educativos españoles han sido capaces de crear o de reorganizar. Adaptaciones curriculares grupales, programas de diversificación, pro- 
gramas de iniciación profesional, aulas enlace, grupos de apoyo, club de lectura, aulas multisensoriales, rincón del ordenador, pizarra digital interactiva, actividades en red, escuela de la experiencia, etc., son algunos de los ejemplos a los que hemos llegado.

Y por último, abiertos al cambio y a los aprendizajes, la experiencia nos enseña que la escuela inclusiva tiene inicio pero no tiene fin, nunca una escuela puede ser considerada totalmente inclusiva, siempre hay algo más por hacer, siempre pueden aparecer nuevas situaciones, los alumnos cambian cada año. La escuela ha de estar preparada para recibir a los alumnos, sin importar la condición de llegada. La escuela se adapta al alumno y no al contrario.

\section{Referências}

AGENCIA EUROPEA PARA EL DESARROLLO DE LA EDUCACIÓN DEL ALUMNADO CON NECESIDADES EDUCATIVAS ESPECIALES. Formación del profesorado para la educación inclusiva: perfil Profesional del Docente en la Educación Inclusiva. Dinamarca, 2012. Disponível em: <https://www.european -agency.org/sites/default/files/te4i-profile-of-inclusive-teachers_Profile-of-Inclusive-Teachers-ES.pdf $>$. Acesso em: 23 abr. 2017.

ALEGRE, O. M. Capacidades docentes para atender la diversidad: una propuesta vinculada a las competencias básicas. Alcalá: Eduforma, 2010.

AYALA, F. El modelo de formación por competencias. 2008. Disponível em: <http://www.modelo.edu.mx/ univ/mcom.ppt>. Acesso em: 16 jun. 2008.

BACHELARD, G. La formation de l'esprit scientifique. París: Vrin, 1996.

BOOTH, T.; AINSCOW, M. Index for Inclusion. Bristol: CSIE, 2000.

ESPAÑA. Ministério de la Presidencia y para las Administraciones Territoriales. Ley Orgánica de Ordenación General del Sistema Edcuativo (LOGSE). Madrid: BOE. Madri, 1990. Disponível em: <https://www.boe. es/diario_boe/txt.php?id=BOE-A-1990-24172>. Acesso em: 23 fev 2017.

FERNÁNDEZ BATANERO, J. M. Competencias docentes y educación inclusiva. Revista Electrónica de Investigación Educativa, Esenada, v. 15, n. 2, p. 82-99, 2013. Disponível em: <http://redie.uabc.mx/vol15no2/ contenido-fdzbatanero.html>. Acesso em: 12 abr. 2017.

HUBERMAN, M. Las fases de la profesión docente. Quaderns digitals, Barcelona, n. 186, p. 5-8, 1989. Disponível em: <http://www.quadernsdigitals.net/datos/hemeroteca/r_12/nr_186/a_2638/2638.html>. Acesso em: 2 abr. 2017.

LE BOTERF, G. De la compétence: essai sur un attracteur étrange, Paris,Les éditions d'organisations, 1994.

MEIRIEU, P. Aprender sí... pero cómo. Barcelona: Octaedro, 1992.

PÉREZ, M. J. La formación permanente del profesorado ante los nuevos retos del sistema educativo universitario. In: Revista Electrónica Interuniversitaria de Formación del Profesorado, 8 (1), Madrid: 2005. p. 1-4

PERRENOUD, P. Diez nuevas competencias para enseñar. Querétaro (México): Ed. Monte Albán, 2004.

SORIANO, Juana.; VIGO, Begońa. La formación de maestros para la atención a la diversidad: evaluación de una experiencia de reflexión conjunta entre futuros maestros de distintas especialidades. In: JORNADAS DE INNOVACIÓN DOCENTE, TECNOLOGÍAS DE LA INFORMACIÓN Y DE LA COMUNICACIÓN E INVESTIGACIÓN EDUCATIVA. 2., 2008, Zaragoza. Anais... Zaragoza: Universidad de Zaragoza, 2008. p. 231-236.

TIMPERLEY, H.; ALTON-LEE, A. Reframing teacher professional learning: an alternative policy approach to strengthening valued outcomes for diverse learners. Review of Research in Education, v. 32, n. 1, p. 328-369, 2008.

ZABALA, A.; ARNAU, L. Cómo aprender y enseñar competencias. Barcelona: Graó. 2007. 


\section{Notas}

${ }^{1}$ Los alumnos una vez realizado el diagnóstico se determina si presentan necesidades específicas de apoyo educativo y a que están ligadas (superdotación, psíquicas, físicas, etc.) y si estas son permanentes o transitorias. Este diagnóstico incluye el tipo de escolarización más adecuado para estos alumnos: centros ordinarios o específicos.

${ }^{2}$ Las Comunidades Autónomas, algo parecido a una configuración de Estado con gobierno propio, tiene las competencias en materia educativa totalmente transferidas desde el 1 de enero de 2000 por lo que el Gobierno de la Nación promulga leyes generales amplias que son desarrolladas por las CCAA.

\section{Correspondência}

Eladio Sebastián-Heredero - Universidade de Alcalá, Departamento de Didatica. C/ Madrid, 1. Sala 3-21, Guadalajara. CEP: 19001. Guadalajara, Espanha.

E-mail: eladio.sebastian@gmail.com

Recebido em 05 de julho de 2017

Aprovado em 11 de agosto de 2017 
